\title{
Classical Initial Conditions for Nucleus-Nucleus Collisions
}

\author{
Yuri V. Kovchegov ${ }^{\text {a* }}$

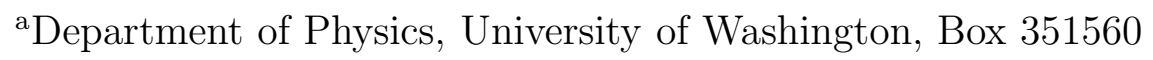 \\ Seattle, WA 98195-1560, USA
}

We present the results of an analytical calculation of the distribution of gluons in the state immediately following a heavy ion collision in the quasi-classical limit of QCD given by McLerran-Venugopalan model. We show that the typical transverse energy $E_{T}$ of the produced gluons is of the order of the saturation scale of the nuclei $Q_{s}$, as predicted by Mueller. We demonstrate that due to multiple rescatterings the obtained gluon distribution remains finite (up to logarithms of $k_{\perp}$ ) in the soft transverse momentum limit of $k_{\perp} \ll Q_{s}$. We compare the gluon spectrum in the single nuclear wave function before the collision to the spectrum of the produced gluons. The total number of produced gluons turns out to be proportional to the total number of gluons inside the nuclear wave function before the collision with the proportionality coefficient $c \approx 2 \ln 2$.

The results presented here are based on reference [1]. Let us consider a collision of two ultrarelativistic nuclei characterized by the saturation scales $Q_{s 1}^{2}$ and $Q_{s 2}^{2}$. We are going to write down an analytical expression for the classical distribution of produced gluons which resums all multiple rescatterings of the gluons with the nucleons in the colliding nuclei. Formally this corresponds to resumming powers of both $Q_{s 1}^{2} / k_{\perp}^{2}$ and $Q_{s 2}^{2} / k_{\perp}^{2}$ [2, 3]. The problem is similar to the problem of finding the classical gluon field of two colliding nuclei formulated in [4], with the exception that here we are interested in the gluon production cross section.

The classical field of two nuclei in the forward light cone has been found in the usual perturbation theory to order $g^{3}$ in [4]. The answer for the gluon multiplicity distribution is the first (lowest order) term of the expansion in powers of $Q_{s 1,2}^{2} / k_{\perp}^{2}$ (see Eq. (4) below) and corresponds in this sense to proton-proton scattering.

A gluon production cross section for a slightly more complicated case of proton-nucleus interactions was derived in [3]. Formally that cross section includes all powers of $Q_{s 1}^{2} / k_{\perp}^{2}$ keeping only the leading power of $Q_{s 2}^{2} / k_{\perp}^{2}$. The calculations in [3] were done in covariant gauge, where multiple final state rescatterings play crucial role in gluon production.

In [1] the same pA process was considered in the framework of light cone perturbation theory in light cone gauge. Knowing the answer for the gluon production cross section from [3] allowed us to determine diagrams which reproduce this answer in the light cone gauge. We demonstrated that an entirely different set of initial state interactions is important

${ }^{*}$ This work has been supported in part by the U.S. Department of Energy under Grant No. DE-FG0397ER41014. 

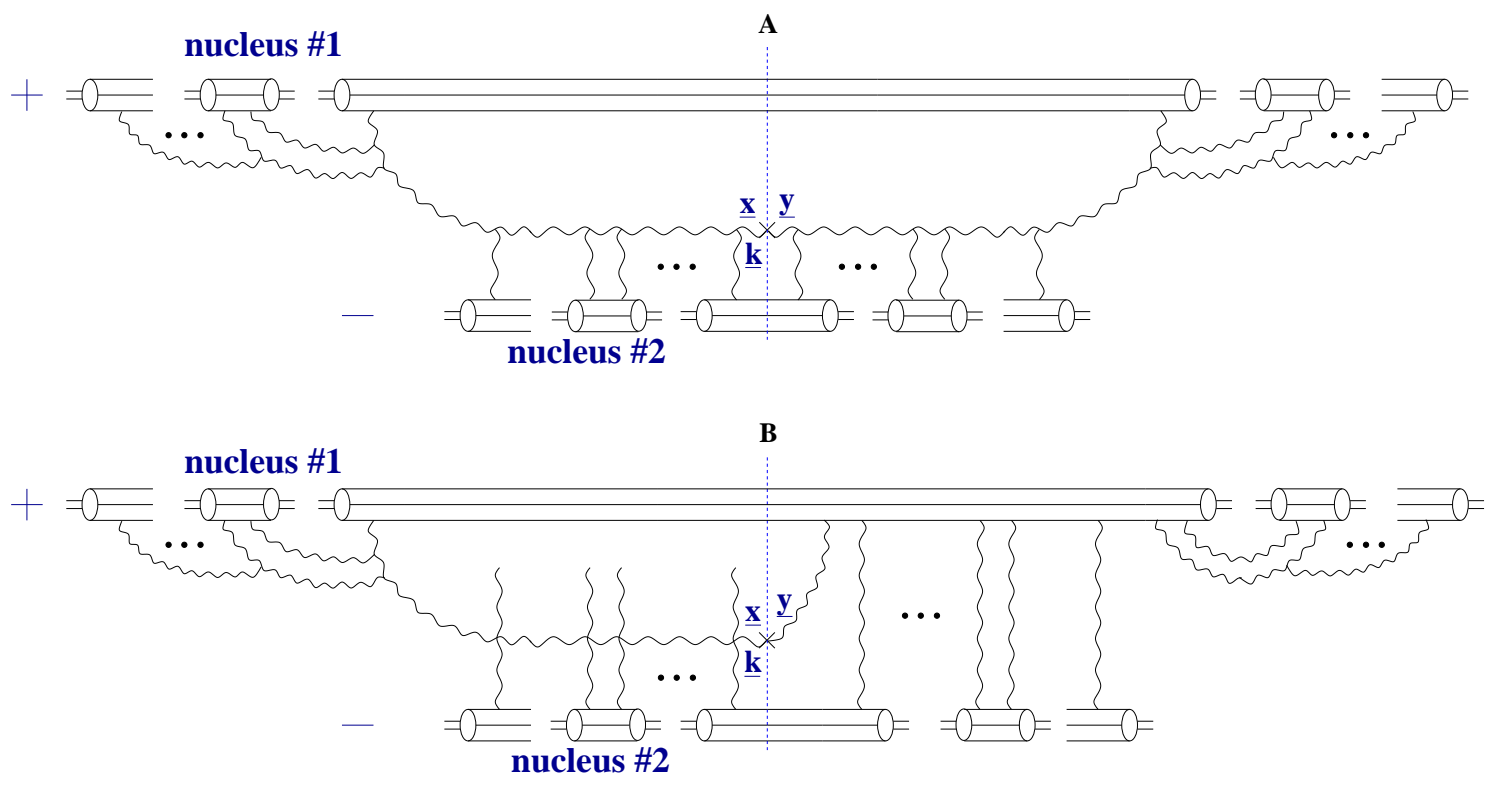

Figure 1. Diagrams contributing to the gluon production in nucleus-nucleus collisions in the $A_{+}=0$ light cone gauge.

there. Generalizing the pA results to the AA case we end up with the diagrams shown in Fig. 1. Summation of the graphs of Fig. 1 1 yields us with the following multiplicity distribution of the produced gluons [1]

$$
\begin{aligned}
& \frac{d N^{A A}}{d^{2} k d^{2} b d y}=\frac{2 C_{F}}{\alpha_{s} \pi^{2}}\left\{-\int \frac{d^{2} z}{(2 \pi)^{2}} e^{i \underline{k} \cdot \underline{z}} \frac{1}{\underline{z}^{2}}\left(1-e^{-\underline{z}^{2} Q_{s 1}^{2} / 4}\right)\left(1-e^{-\underline{z}^{2} Q_{s 2}^{2} / 4}\right)+\right. \\
& +\int \frac{d^{2} x d^{2} y}{(2 \pi)^{3}} e^{i \underline{k} \cdot(\underline{x}-\underline{y})} \frac{\underline{x}}{\underline{\underline{x}}{ }^{2}} \cdot \frac{\underline{y}}{\underline{y}^{2}}\left[\frac{1}{\underline{x}^{2} \ln \frac{1}{\mid \underline{x \mid \mu}}}\left(1-e^{-\underline{x}^{2} Q_{s 1}^{2} / 4}\right)\left(1-e^{-\underline{x}^{2} Q_{s 2}^{2} / 4}\right)+\right. \\
& \left.\left.+\frac{1}{\underline{y}^{2} \ln \frac{1}{|\underline{\mid}| \mu}}\left(1-e^{-\underline{y}^{2} Q_{s 1}^{2} / 4}\right)\left(1-e^{-\underline{y}^{2} Q_{s 2}^{2} / 4}\right)\right]\right\} .
\end{aligned}
$$

Eq. (11) is our main result. It provides us with the number of gluons produced in a heavy ion collision per unit transverse momentum phase space, per unit rapidity interval at the given impact parameter $b$ yielding the initial conditions for possible thermalization of the gluonic system at later times.

To explore the properties of the distribution (11) we first perform the transverse coordinate integration in the logarithmic approximation (see [1]), which would transform it into the following simplified form

$$
\frac{d N^{A A}}{d^{2} k d^{2} b d y}=\frac{C_{F}}{\alpha_{s} 2 \pi^{3}} \frac{1}{\underline{k}^{2}}\left[\left(Q_{s 1}^{2}+Q_{s 2}^{2}\right) e^{-\frac{\underline{k}^{2}}{Q_{s 1}^{2}+Q_{s 2}^{2}}}-Q_{s 1}^{2} e^{-\frac{k^{2}}{Q_{s 1}^{2}}}-Q_{s 2}^{2} e^{-\frac{\underline{k}^{2}}{Q_{s 2}^{2}}}\right]
$$




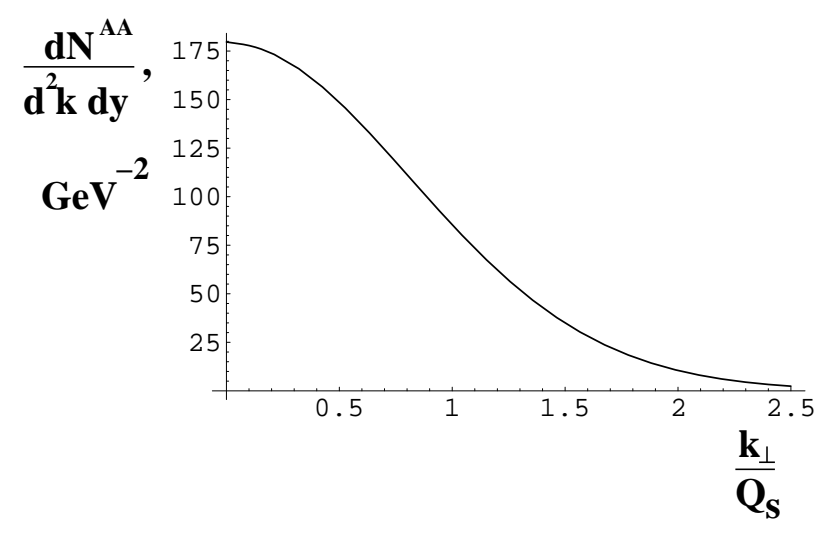

Figure 2. Distribution of the produced gluons given by Eq. (2) for a central AA collision as a function of $k / Q_{s}$ with the transverse area of the nuclei being $S_{\perp}=50 \mathrm{fm}^{2}$. The approximation of Eq. (22) is valid only for $k_{\perp} \lesssim Q_{s}$.

The distribution of Eq. (2) is plotted in Fig. 2 as a function of $k / Q_{s}$ for the case of two identical cylindrical nuclei with $Q_{s 1}=Q_{s 2}=Q_{s}$ and with the cross sectional area $S_{\perp}=\pi R^{2} \approx 50 \mathrm{fm}^{2} \approx 1250 \mathrm{GeV}^{-2}$. Note that Eq. (2) is valid only in the not very large transverse momentum region $k_{\perp} \lesssim Q_{s}$ [1].

As one can see, the distribution in Fig. 2 remains finite as $k_{\perp} / Q_{s} \rightarrow 0$. If one takes the $k_{\perp} \ll Q_{s}$ limit of Eq. (2) then the distribution goes to a constant

$\frac{d N^{A A}}{d^{2} k d^{2} b d y} \rightarrow \frac{C_{F}}{\alpha_{s} 2 \pi^{3}} \quad$ as $\quad \frac{k_{\perp}}{Q_{s}} \ll 1$.

This means that the exact expression of Eq. (1) may only have logarithmic divergences in the infrared limit. Therefore, finiteness of Eq. (2) at small transverse momenta demonstrates that multiple rescatterings are the reason the hadronic and nuclear single particle inclusive production cross sections remain finite in the soft momentum region.

Since the distribution of Eq. (1) includes the lowest order in $\alpha_{s}$ diagrams in it, one readily derives that in the $k_{\perp} / Q_{s} \rightarrow \infty$ limit the distribution falls off as $1 / k_{\perp}^{4}$ 四]

$\frac{d N^{A A}}{d^{2} k d^{2} b d y} \sim \frac{Q_{s 1}^{2} Q_{s 2}^{2}}{\alpha_{s} \underline{k}^{4}} \quad$ as $\quad \frac{k_{\perp}}{Q_{s}} \rightarrow \infty$.

The average transverse energy per produced gluon given by Eq. (2) for the case of two identical nuclei with $Q_{s 1}=Q_{s 2}=Q_{s}$ is

$$
<E_{T}>=\frac{\sqrt{\pi}(\sqrt{2}-1)}{\ln 2} Q_{s} \approx 1.06 Q_{s} .
$$

That is, the typical transverse energy of the produced gluons is of the order of the saturation scale, as was previously conjectured by Mueller in [0]. The saturation scale for a large nucleus scales as $Q_{s}^{2} \sim A^{1 / 3}$ with atomic number justifying the use of the small coupling expansion for large nuclei [2,5]. 


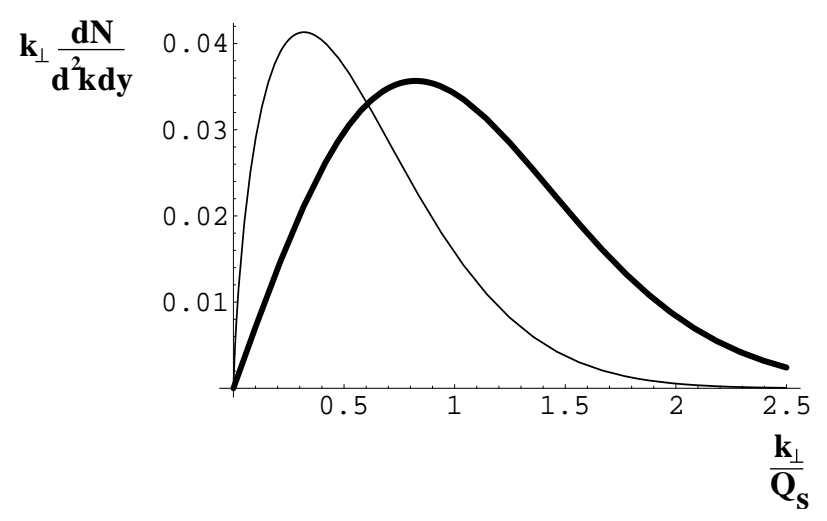

Figure 3. The gluon distribution as a function of $k_{\perp} / Q_{s}$ in one of the nuclei before the collision (thin line) and the gluon distribution after the collision (thick line).

Fig. 3 compares the transverse momentum distribution of gluons in the single nuclear wave function before the collision (thin line) with the distribution of produced gluons after the collision (thick line). One can see that the typical transverse momentum of gluons gets broadened due to multiple rescatterings in the collision. The total number of gluons produced in the collision can be found by integrating Eq. (21) over $k_{\perp}$. The result yields for two identical nuclei

$\frac{d N^{A A}}{d^{2} b d y}=\frac{C_{F} Q_{s}^{2} \ln 2}{\alpha_{s} \pi^{2}}$.

The total number of produced gluons turns out to be proportional to the total number of gluons in the wave function of a single nucleus before the collision [1] with the proportionality coefficient

$c=2 \ln 2 \approx 1.39$.

The obtained value for the "gluon liberation" coefficient $c=2 \ln 2$ is close to one, as was originally suggested by Mueller [5].

\section{REFERENCES}

1. Yuri V. Kovchegov, NT-UW-00-031, hep-ph/0011252.

2. L. McLerran and R. Venugopalan, Phys. Rev. D 49, 2233 (1994); 49, 3352 (1994); 50, 2225 (1994); Yu.V. Kovchegov, Phys. Rev. D 54, 5463 (1996); 55, 5445 (1997); J. Jalilian-Marian, A. Kovner, L. McLerran, and H. Weigert, Phys. Rev. D 55, 5414 (1997).

3. Yu. V. Kovchegov, A.H. Mueller, Nucl. Phys. B529, 451 (1998).

4. A. Kovner, L. McLerran, and H. Weigert, Phys. Rev. D 52, 6231 (1995); 52, 3809 (1995); Yu.V. Kovchegov, D. H. Rischke, Phys. Rev. C 56, 1084 (1997); M. Gyulassy, L. McLerran, Phys. Rev. C 56, 2219 (1997).

5. A.H. Mueller, Nucl. Phys. B572, 227 (2000). 